\title{
Optimization of PAPR of 8\&16 QPSK using MIMO - OFDM in AWGN channel Through Grey code input bit stream
}

\author{
Sathish Kumar Yegireddi, Vemakoti viveknand
}

\begin{abstract}
Orthogonal Frequency Division Multiplexing (OFDM) is an essential part in $5 \mathrm{G}$ communication which is an efficient method of data transmission. It is a base for high speed communication systems. Though this technique is helpful to mobile communication, satellite communication and some other like which are part of digital communication, it has few drawbacks in that mainly suffers from high Peak-to-Average Power Ratio (PAPR). Due to high PAPR there is inefficient use of high power amplifier and this could limit transmission efficiency, high DC power consumption In addition to a drain on battery life, high power consumption has other effects on the cost of the design. For instance, the power supply unit becomes more expensive. And, in order to dissipate high peak transmit powers of 3.5 W or higher, heat dissipation techniques (such as a large heat sink and special board designs) will increase overall system cost and form factor. Finally, semiconductor manufacturers will have to use more expensive packaging designed to sustain high power consumption, thereby increasing the component cost.

In other word OFDM consist of large number of independent subcarriers, as a result of which the amplitude of such a signal can have high peak values. In this project, we introduce modified OFDM techniques using LDPC and TURBO codes to reduce PAPR. The simulation results show PAPR can be reduced by applying the proposed technique. In this project we consider AWGN channel.
\end{abstract}

\section{Index Terms- OFDM,LDPC,TURBO, AWGN}

\section{INTRODUCTION}

Orthogonal Frequency Division Multiplexing (OFDM) is an essential part in $5 \mathrm{G}$ communication which is an efficient method of data transmission. It is a base for high speed communication systems. Though this technique is helpful to mobile communication, satellite communication and some other like which are part of digital communication, it has few drawbacks in that mainly suffers from high Peak-to-Average Power Ratio (PAPR). Due to high PAPR there is inefficient use of high power amplifier and this could limit transmission efficiency, high DC power consumption In addition to a drain on battery life, high power consumption has other effects on

Sathish Kumar Yegireddi M.Tech (Embedded System) Department of ECE,IIIT Srikakulam, RAJIV GANDHI UNIVERSITY OF KNOWLEDGE TECHNOLOGIES(RGUKT),Andhra Pradesh, India.

Vemakoti Viveknand M.Tech, MBA, is a curious researcher in quantum electrodynamics and high speed signal transmissions though had deep insights on photovoltaic cells' performance calibrations in his previous research papers. the cost of the design. For instance, the power supply unit becomes more expensive. And, in order to dissipate high peak transmit powers of $3.5 \mathrm{~W}$ or higher, heat dissipation techniques (such as a large heat sink and special board designs) will increase overall system cost and form factor. Finally, semiconductor manufacturers will have to use more expensive packaging designed to sustain high power consumption, thereby increasing the component cost. In other word OFDM consist of large number of independent subcarriers, as a result of which the amplitude of such a signal can have high peak values. In this project, we introduce modified OFDM techniques using LDPC and TURBO codes to reduce PAPR. The simulation results show PAPR can be reduced by applying the proposed technique. In this project we consider AWGN channel.

\section{INTRODUCTION TO 6G TECHNOLOGY}

\section{A. $6 G$ intro}

Wireless mobile communication is being used from many years, but day by day Need of facilities on mobile is increasing, so time to time next integrated Versions of network is introduced. There are various generations of mobile Network 1G, 2G, 3G, 4G and 5G. Latest version is $5 \mathrm{G}$, but only some countries are using $5 \mathrm{G}$ wireless network. $5 \mathrm{G}$ network is strong and very fast wireless communication network, it is and it will fulfill most of the requirement of users. But it is not end of the desires, so for meet desires of user next generation of mobile network also introduced $6 \mathrm{G} \&$ 7G. This paper is about introduction and advancement of $6 \mathrm{G}$ $\& 7 \mathrm{G}$ for future.

Today the whole world is aware of the revolutionary changes in cell phone communication field. Wireless communication has brought in the new innovation in this field. In the context of present scenario the $3 \mathrm{G}$ experienced better internet experience. Later on $3 \mathrm{~g}$ has been improvised. It has been felt the urgency to have a better communication networks then $5 \mathrm{~g}$ has came which can be a complete wireless communication without any hindrance and limitations. It is completely advanced in terms of wireless communication. In 5G system each and every cell phone will have a permanent home "IP address and care of address .Now awaiting future 
will experience 6G. In present time cell phones have everything and are compact, with high memory and high speed with low power consumption. Today Bluetooth technology and other technology are just like a child's play. 6G wireless cell phone communication network shall meet world class standard covering the whole world under its communication just like Global covering system has been devised by some companies.

This individual system creates difficulty in space roaming. $7 \mathrm{G}$ mobile phone communication system is developed to integrate these in one unit communication system. $6 \mathrm{G}$ means 6th generation wireless mobile technology.

\section{B. Necessity of $6 G$}

6G technology increase performance and maximize our data throughput. This technology also provide more security to our system and data, it also expand our data configuration options. In this technology wireless broadband will use to connect device to internet. Data speed of $6 \mathrm{G}$ devices will be $1 \mathrm{~GB}$ or even more. $6 \mathrm{G}$ technology also consists of better security of wireless standard and data transmission. 6G technologies will more than the expectation of the users. Because, 6G is satellite based network so roaming and handover from one satellite to another satellite will be issue that will be solved soon.

\section{Figures}

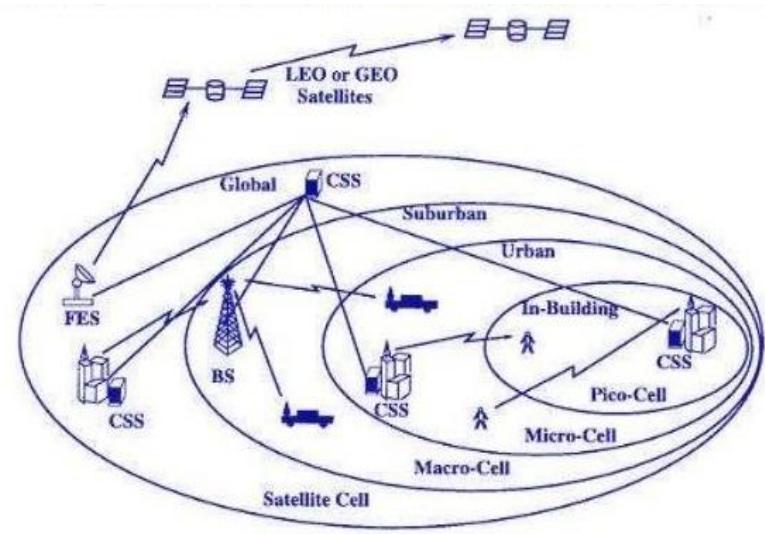

Figere.1.Satellite network for $6 \mathrm{G}$

\section{Overview}

We can say that, present wireless technology (1G to $4 \mathrm{G})$ is meeting all the requirements of users. But present generation wants everything should be fast that's why we are thinking about broad and fast across all the boundary of requirement and efficiency. That's why we are thinking about next generation of wireless network $6 \mathrm{G}$.

\section{NAKAGAMI FADING CHANNEL DISTRIBUTION}

The Nakagami distribution or the Nakagami-m distribution is a probability distribution related to the gamma distribution.
The family of Nakagami distributions has two parameters: a shape parameter and a second parameter controlling spread.

Based on the approximated expression of the Q-function by Chiani, Dardari, and Simon, a simple and accurate generalized closed-form expression for the bit error rate (BER) over Nakagami-m fading channel is derived. The expression is given as a product of the well-known gamma function and a finite sum of functions of the Nakagami -m fading parameter. The derived expression is applicable for any real $\geq 0.5$, and for any coherent modulation scheme of any order. Numerical results are used to validate the derived expressions using different combinations of modulation orders and Nakagami-m fading parameters.

\section{A. Simulation result}

The probability of the error expression derived in (16) can be used now to evaluate the performance in terms of BER over Rayleigh fading channel $(\mathrm{m}=1)$ as well as Nakagami-m fading channel for any modulation scheme by simply choosing the appropriate values. Testing the expression derived is conducted using three different scenarios. First, we choose a modulation scheme from Table I (Binary Phase Shift Keying) and test the performance over different values of Nakagami-m fading parameters, specifically $m=1,2,3$ and 4 . Numerical results for equation which represent exact BER curve were compared with the approximated BER curve that has been evaluated using the expression.

The results are shown in Following Figure. It can be seen that the approximated expression is highly accurate and matches the numerical results for different values of $\mathrm{m}$ as well as different values of $y$.

The independent observations $\boldsymbol{X}_{\mathbf{1}}=\boldsymbol{x}_{\mathbf{1}},----\boldsymbol{X}_{\boldsymbol{n}}=\boldsymbol{x}_{\boldsymbol{n}}$ from the Nagakami distribution, the likelihood function is

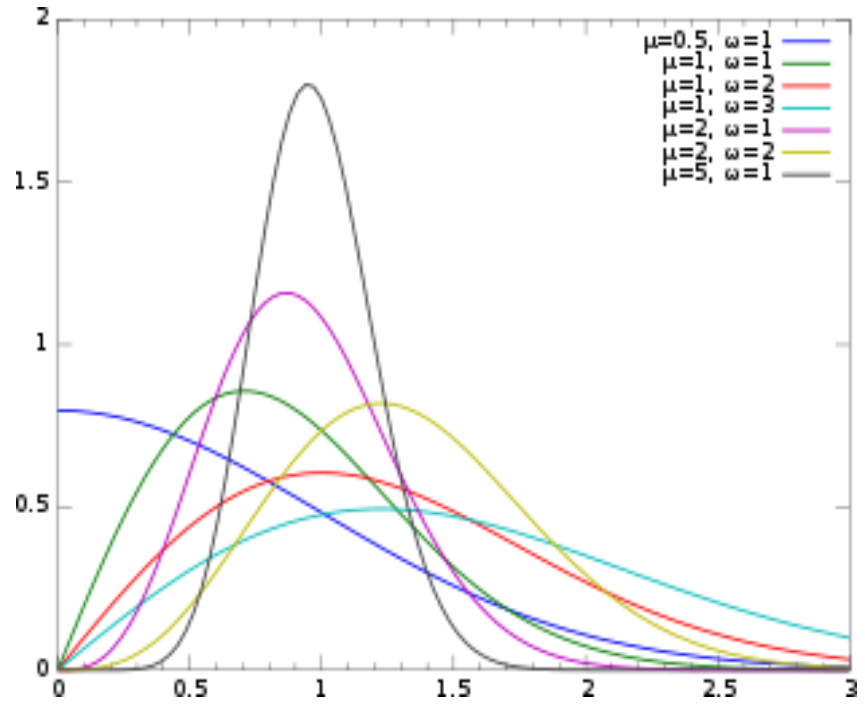

Figer.2: Waveforms Of Distributed Channel 


\section{B. Probability density function:}

A probability density function (PDF), or density of a continuous random variable, is a function whose value at any given sample (or point) in the sample space (the set of possible values taken by the random variable) can be interpreted as providing a relative likelihood that the value of the random variable would equal that sample

Pdf : $\left.\mathbf{f}\left(\mathbf{x}_{j}^{*} \mathbf{m}_{w} \Omega\right)=\frac{2 m^{m}}{f(m) \Omega^{m}} x^{2 m-1} \exp \left(\frac{m}{\Omega}\right) x^{2}{ }_{a}\right), \forall \boldsymbol{x} \geq \mathbf{0}$,

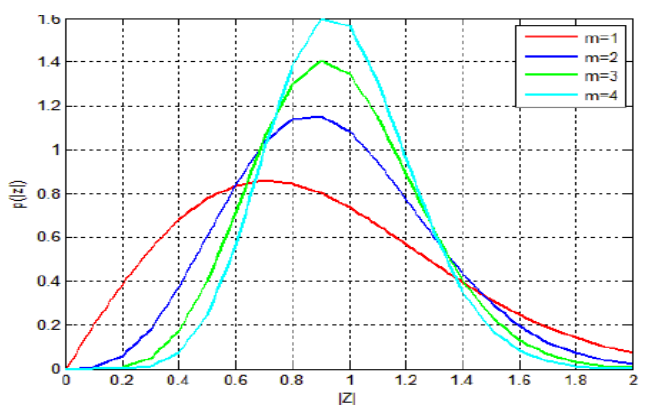

Fig ure.no.3:Waveform of PDF with varying $m$ values

\section{Quadriphase psk,4-psk,or 4-qam}

Sometimes this is known as quadriphase PSK, 4-PSK, or 4-QAM. (Although the root concepts of QPSK and 4-QAM are different, the resulting modulated radio waves are exactly the same.) QPSK uses four points on the constellation diagram, equispaced around a circle. With four phases, QPSK can encode two bits per symbol, shown in the diagram with Gray coding to minimize the bit error rate (BER) - sometimes misperceived as twice the BER of BPSK.

The mathematical analysis shows that QPSK can be used either to double the data rate compared with a BPSK system while maintaining the same bandwidth of the signal, or to maintain the data-rate of BPSK but halving the bandwidth needed. In this latter case, the BER of QPSK is exactly the same as the BER of BPSK - and deciding differently is a common confusion when considering or describing QPSK. The transmitted carrier can undergo numbers of phase changes.

Given that radio communication channels are allocated by agencies such as the Federal Communication Commission giving a prescribed (maximum) bandwidth, the advantage of QPSK over BPSK becomes evident: QPSK transmits twice the data rate in a given bandwidth compared to BPSK - at the same BER. The engineering penalty that is paid is that QPSK transmitters and receivers are more complicated than the ones for BPSK. However, with modern electronics technology, the penalty in cost is very moderate. As with BPSK, there are phase ambiguity problems at the receiving end, and differentially encoded QPSK is often used in practice.

\section{Block diagram OF QPSK}

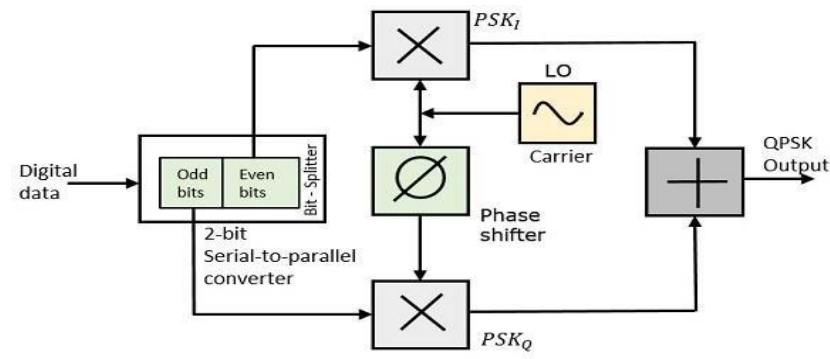

Figure no.4: Block Diagram Of QPSK

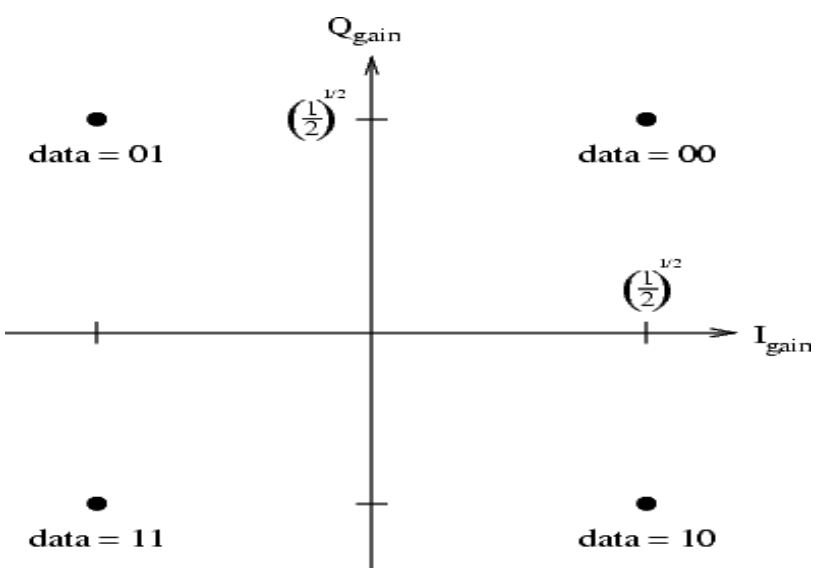

Figure no.5: constellation diagram

\section{CODING TECHNIQUES}

\section{A. Cyclic prefix:}

In telecommunications, the term cyclic prefix refers to the prefixing of a symbol, with a repetition of the end. The receiver is typically configured to discard the cyclic prefix samples, but the cyclic prefix serves two purposes:

It provides a guard interval to eliminate intersymbol interference from the previous symbol.

It repeats the end of the symbol so the linear convolution of a frequency-selective multipath channel can be modeled as circular convolution, which in turn may transform to the frequency domain via a discrete Fourier transform. This approach accommodates simple frequency domain processing, such as channel estimation and equalization.

For the cyclic prefix to serve its objectives, it must have a length at least equal to the length of the multipath channel. The concept of a cyclic prefix is traditionally associated with OFDM systems, however the cyclic prefix is now also used in 
single carrier systems to improve the robustness to multipath propagation.

\section{Principle:}

A cyclic prefix is often used in conjunction with modulation to retain sinusoids' properties in multipath channels. It is well known that sinusoidal signals are eigenfunctions of linear, and time-invariant systems. Therefore, if the channel is assumed to be linear and time-invariant, then a sinusoid of infinite duration would be an eigenfunction. However, in practice, this cannot be achieved, as real signals are always time-limited. So, to mimic the infinite behavior, prefixing the end of the symbol to the beginning makes the linear convolution of the channel appear as though it were circular convolution, and thus, preserve this property in the part of the symbol after the cyclic prefix.

\section{B. Use in OFDM:}

OFDM uses cyclic prefixes to combat multipath by making channel estimation easy. As an example, consider an OFDM system that has subcarriers. The message symbol can be written as:

$$
d=\left[d_{0}, d_{1}, \ldots d_{N-1}\right]^{T}
$$

The OFDM symbol is constructed by taking the inverse discrete Fourier transform (IDFT) of the message symbol, followed by a cyclic prefixing. Let the symbol obtained by the IDFT be denoted by

$$
X^{1}=[x[0], x[1], \ldots, x[N]]^{T}
$$

\section{Channel Coding}

Channel coding, also known as forward error control coding (FECC), is a process of detecting and correcting bit errors in digital communication systems. Channel coding is performed both at the transmitter and at the receiver. At the transmit side, channel coding is referred to as encoder, where extra bits (parity bits) are added with the raw data before modulation. At the receive side, channel coding is referred to as the decoder. Channel coding enables the receiver to detect and correct errors, if they occur during transmission due to noise, interference and fading. This book presents the salient concepts, underlying principles and practical realization of channel coding schemes, as listed below:

- channel coding techniques for wireless communications such as

- linear convolution codes

- turbo codes,

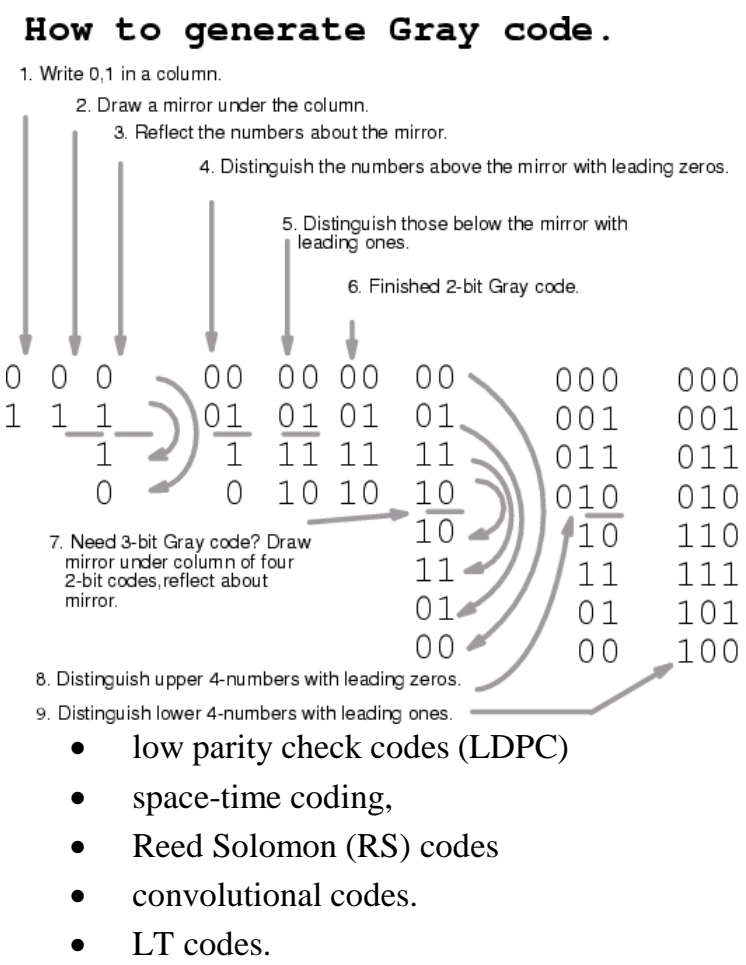

Source Coding:

Entropy of a source is the measure of information. Basically, source codes try to reduce the redundancy present in the source, and represent the source with fewer bits that carry more information.

Data compression which explicitly tries to minimize the average length of messages according to a particular assumed probability model is called entropy encoding.

Various techniques used by source coding schemes try to achieve the limit of Entropy of the source. $C(x) \geq H(x)$, where $H(x)$ is entropy of source (bitrate), and $C(x)$ is the bitrate after compression. In particular, no source coding scheme can be better than the entropy of the source.

1) Source coding techniques:

- Huffman Code.

- Lemple-Ziv Code.

- Shannon fanon Code.

- Arithmetic Code.

\section{Gray code:}

The reflected binary code (RBC), also known just as reflected binary (RB) or Gray code after Frank Gray, is an ordering of the binary numeral system such that two successive values differ in only one bit (binary digit). The reflected binary code was originally designed to prevent spurious output from electromechanical switches. Today, Gray codes are widely 
used to facilitate error correction in digital communications such as digital terrestrial television and some cable TV systems.

Bell Labs researcher Frank Gray introduced the term reflected binary code in his 1947 patent application, remarking that the code had "as yet no recognized name". He derived the name from the fact that it "may be built up from the conventional binary code by a sort of reflection process".

The code was later named after Gray by others who used it. Two different 1953 patent applications use "Gray code" as an alternative name for the "reflected binary code". one of those also lists "minimum error code" and "cyclic permutation code" among the names. A 1954 patent application refers to "the Bell Telephone Gray code". Other names include "cyclic binary code" and "cyclic progression code". It is also named as "Unit Distance Code " as any two adjacent codes in this set is differ by one
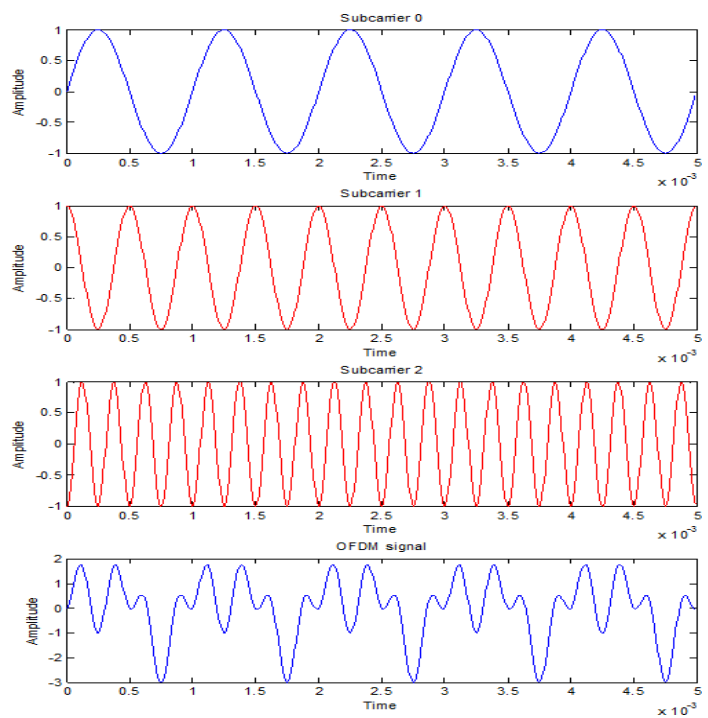

Figure no5: Step by step process of Gray Code

\section{PAPR}

\section{A. Definition}

The peak-to-average power ratio (PAPR) is the peak amplitude squared (giving the peak power) divided by the $\underline{\mathrm{RMS}}$ value squared (giving the average power). It is the square of the crest factor.

$$
\begin{gathered}
P A P R=\frac{\left|x_{\text {paak }}\right|^{2}}{x_{\text {rms }}{ }^{2}}=C^{2} \\
P A P R=10 \log _{10} \frac{\left|x_{\text {peak }}\right|^{2}}{x_{\text {rms }}{ }^{2}}=C_{d B}
\end{gathered}
$$

\section{B. PAPR Reduction}

Crest factor and PAPR are therefore dimensionless quantities. While the crest factor is defined as a positive real number, in commercial products it is also commonly stated as the ratio of two whole numbers, e.g., 2:1. The PAPR is most used in signal processing applications. As it is a power ratio, it is normally expressed in decibels $(\mathrm{dB})$. The crest factor of the test signal is a fairly important issue in loudspeaker testing
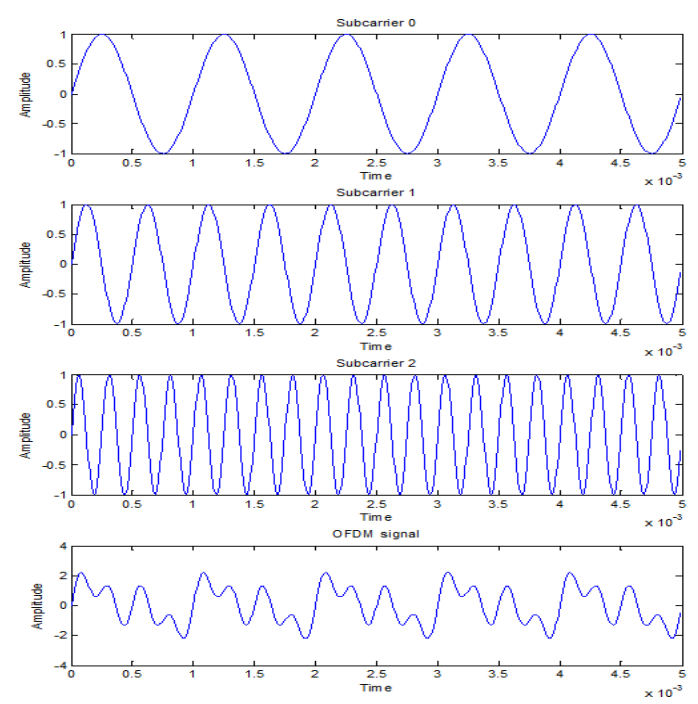

Figure.no.6: Different waveforms of PAPR

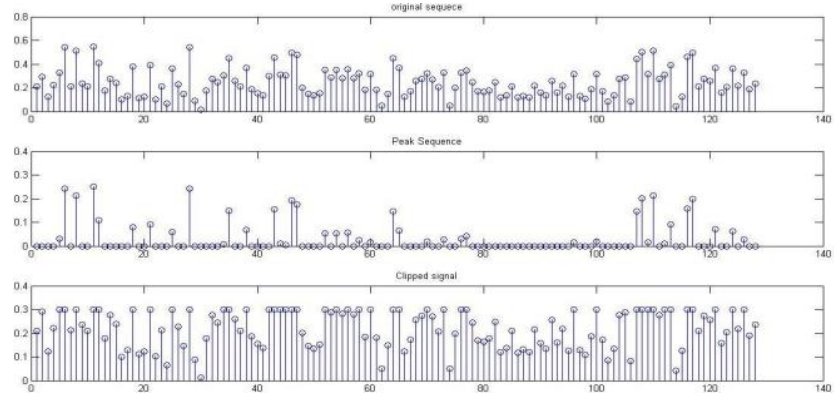

Figure.no.7: Different waveforms of PAPR

\section{PAPR on the performance of mimo ofdm system}

The peak to average power ratio (PAPR) of a transmitted signal is one of main challenges in wideband multi-carrier systems that use orthogonal frequency division multiplexing (OFDM) or multiple-input multiple-output (MIMO) OFDM. Understanding the effects of PAPR on OFDM and MIMO-OFDM systems is critical when determining what techniques to use improve system performance. For the purposes of this blog post, we can use the terms OFDM and MIMO-OFDM interchangeably without affecting the meaning of PAPR. 


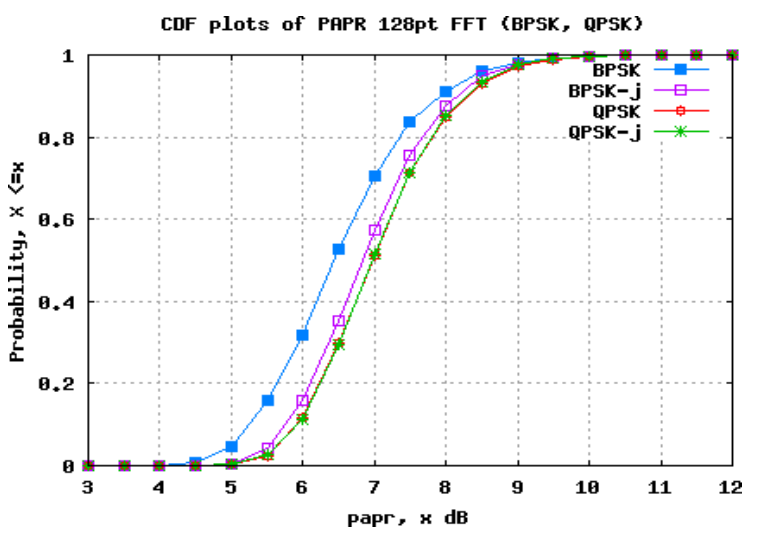

Figure.no.8:Different waveforms of PAPR

\section{Optimization of PAPR}

Optimizing OFDM signals based on convex optimization algorithm for PAR reduction was evaluated on a RF PA. It showed a limited improvement in power performance near compression; an extra $1 \mathrm{~dB}$ in output power and $3.4 \%$ in efficiency. While in the backed-of region it had 5.4\% PAE improvement and a gain of $5 \mathrm{~dB}$ in output power. A reason for such behavior is the increase in lower peak density distribution in the signal. Spectral emission was considered to be a draw back in the method which needs further investigations in the algorithm in order to meet the standard requirements for emission. The main cause to the spectral leakage is the absence of the power free-subcarriers. It is advised to adjust the minimization algorithm to consider part of the sub-carriers as guard intervals.

\section{E. PAPR in mimo-ofdm system:}

The use of a large number of subcarriers introduces a high PAPR in OFDM systems. PAPR can be defined as the relationship between the maximum power of a sample in a transmit OFDM symbol and its average power.

$$
P A P R=10 \log _{10} \frac{\left|x_{\text {peak }}\right|^{2}}{x_{\text {rms }}^{2}}=C_{d B}
$$

Where $P$ peak and $P$ average are the peak and average power of a given OFDM symbol.

The same definition of PAPR is applied to MIMO-OFDM systems. A high PAPR appears when a number of subcarriers of a given OFDM symbol are out of phase with each other. Figure 9 shows the time domain representation of the 3 subcarriers of an OFDM symbol. The right column indicates that the subcarriers are out of phase, which causes an increase in PAPR of about $2.5 \mathrm{~dB}$ compared to the subcarriers in the left column. Depending on the out-of-phase amount per subcarrier, the PAPR can vary up to its theoretically maximum of $10 \log _{10}(\mathrm{~N})(\mathrm{dB})$, where $\mathrm{N}$ is the number of subcarriers. In Figure 9, the 3 subcarriers reach their minimum amplitude at a same time, causing a large negative overshoot in the resulting composite OFDM signal.
Moving wireless local area networks (WLANs) to the 5-GHz band is very appealing because of the leap in data rates from 11 or $22 \mathrm{Mb} / \mathrm{s}$ to $54 \mathrm{Mb} / \mathrm{s}$. But, making the leap to 5-GHz also means the use of orthogonal frequency division multiplexing (OFDM), which comes with its share of challenges.

OFDM is frequently referred to as multi-carrier modulation because it transmits signals over multiple subcarriers simultaneously, enabling it to boost WLAN data rates up to $54 \mathrm{Mb} / \mathrm{s} .1$ It is based on the Fast Fourier transform (FFT) concept, which allows the multiple subcarriers to overlap yet maintain their integrity. 2 Because these subcarriers are sent at lower data rates,

OFDM systems perform robustly in severe multi-path environments. As a result, this modulation approach is being widely adopted in 5-GHz WLAN implementations.

High throughput data rates are achieved in OFDM due to precise carrier spacing and exact amplitude and phase settings for each individual carrier constellation. This is accomplished using computational modulation schemes rather than traditional analog modulation. OFDM's resistance to multi-path interference results from the increased symbol duration for each individual carrier (as compared to other modulation schemes with the same data throughput) and from the use of a cyclic prefix (guard interval) preceding each symbol.

When considering the overall engineering of an OFDM modem, the design of the digital baseband processor (BBP) has many benefits, and few, if any, drawbacks. However, from the perspective of the RF section, traditional OFDM modem designs face a number of key design issues that impact system cost and performance which must be overcome in order to deliver an industry-leading WLAN modem. These include power consumption, linearity, image rejection, phase distortion and phase noise. If these issues are not resolved, the resulting 5-GHz WLAN modem will deliver lower than anticipated data rates and compromised range.

Below we will explore each of these topics and talk about potential solutions to these problems. Let's start with peak-to-average power ratios.

\section{F. power peaks:}

One of the most difficult engineering concerns in the RF portion of traditional OFDM modems is handling very large peak-to-average power ratios (PAPRs). A peak in the signal power will occur when all, or most, of the sub-carriers align themselves in phase. In general, this will occur once every symbol period 


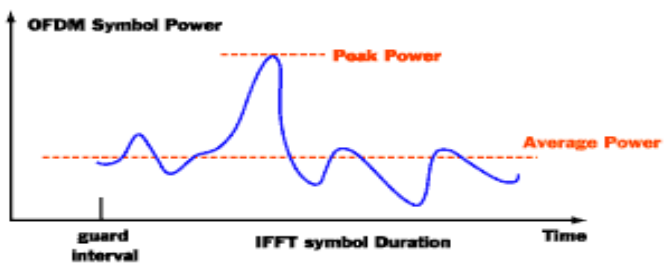

Figure no9:OFDM peak-to-Average Power Ratio Over one symbol Interval

The value of the PAPR is directly proportional to the number of carriers, and is given by:

$$
\operatorname{PAPR}(\mathrm{dB})=10 \log (\mathrm{N})
$$

where $\mathrm{N}$ is the number of carriers.

For example, for the 802.11a OFDM standard, a PAPR of 17 $\mathrm{dB}$ results if the phases of all 52 carriers line up during a symbol period. In order to accommodate this peak in power, the radio's RF power amplifier (PA) must provide gain without compression for every peak power level. In other words, the PA will provide less RF power between peaks, by an amount given by the PAPR.

Not only does this imply that the power amplifier must be oversized in terms of its average power requirement, it also means that the efficiency of the PA will suffer dramatically, since its DC power consumption is determined by the peak power level. Since the DC power consumption of the PA represents a significant portion of the total DC power for a radio, traditionally designed OFDM modems are not power efficient

To put this in perspective, the maximum power efficiency of a Class B PA is $78.5 \%$. However, when accounting for a signal having a PAPR of $10 \mathrm{~dB}$, this efficiency drops to $7.85 \%$. Hence, to achieve a power level of $100 \mathrm{~mW}$ will require a DC power consumption of $1.3 \mathrm{~W}$. This very high DC power consumption limits the attractiveness of OFDM modulation for portable applications, including 5-GHz WLAN enabled devices such as PC laptops and personal digital assistants (PDAs).

\section{More PAPR problems:}

In addition to creating design problems for the PA, the high peak-to-average power ratio of the OFDM modulation scheme also requires highly linear up converters. This means that the up converter must have a high-level compression point, which also results in high DC power consumption.
In addition to a drain on battery life, high power consumption has other effects on the cost of the design. For instance, the power supply unit becomes more expensive. And, in order to dissipate high peak transmit powers of $3.5 \mathrm{~W}$ or higher, heat dissipation techniques (such as a large heat sink and special board designs) will increase overall system cost and form factor. Finally, semiconductor manufacturers will have to use more expensive packaging designed to sustain high power consumption, thereby increasing the component cost.

To meet consumer expectations for user autonomy (long battery life in portable applications), OEM manufacturers are specifying low power consumption as part of their system requirements. But, merely specifying a change does not solve the problem. The technical challenge, then, is before the OFDM modem designers who must achieve acceptable levels of power consumption in their designs. Until then, the penetration of WLANs using OFDM will likely be limited to high-end, low-volume products.

\section{PAPR SIMULATION ON RESULT}

Here the following are constellation diagrams and papr.

\section{A. 8QPSK Simulation}

The submitting author is responsible for obtaining agreement of all coauthors and any consent required from sponsors before submitting a paper. It is the obligation of the authors to cite relevant prior work.
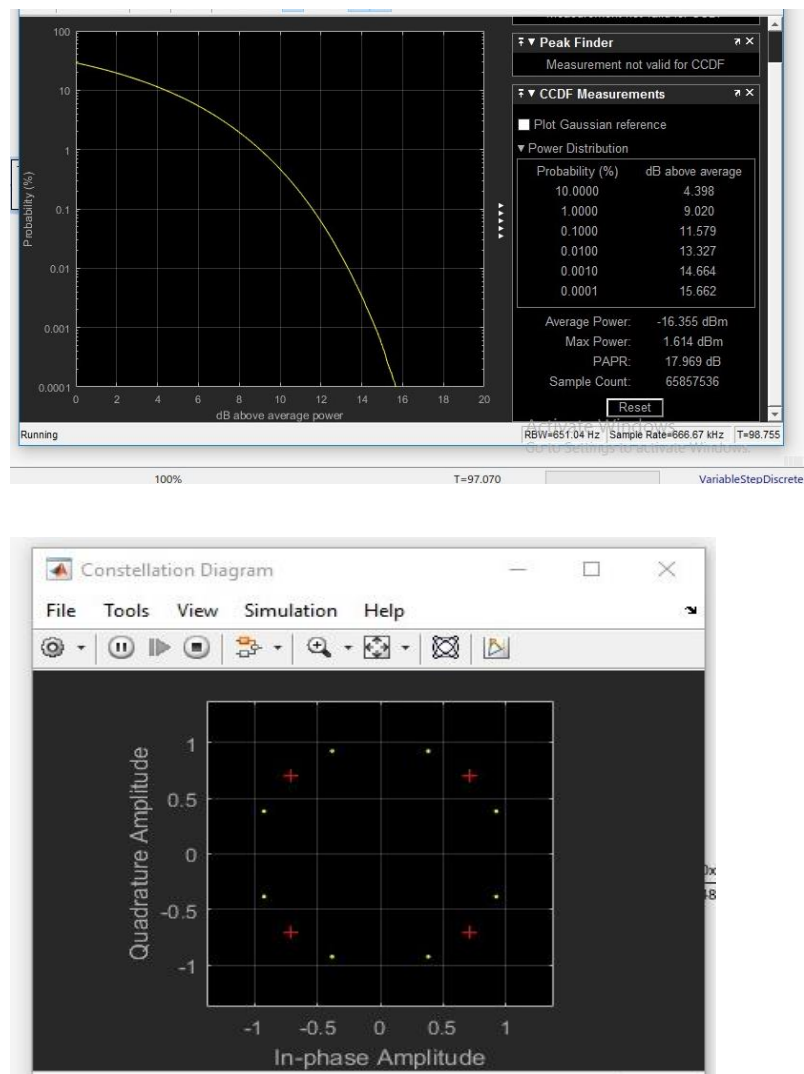


\section{B. 64QPSK Simulation}
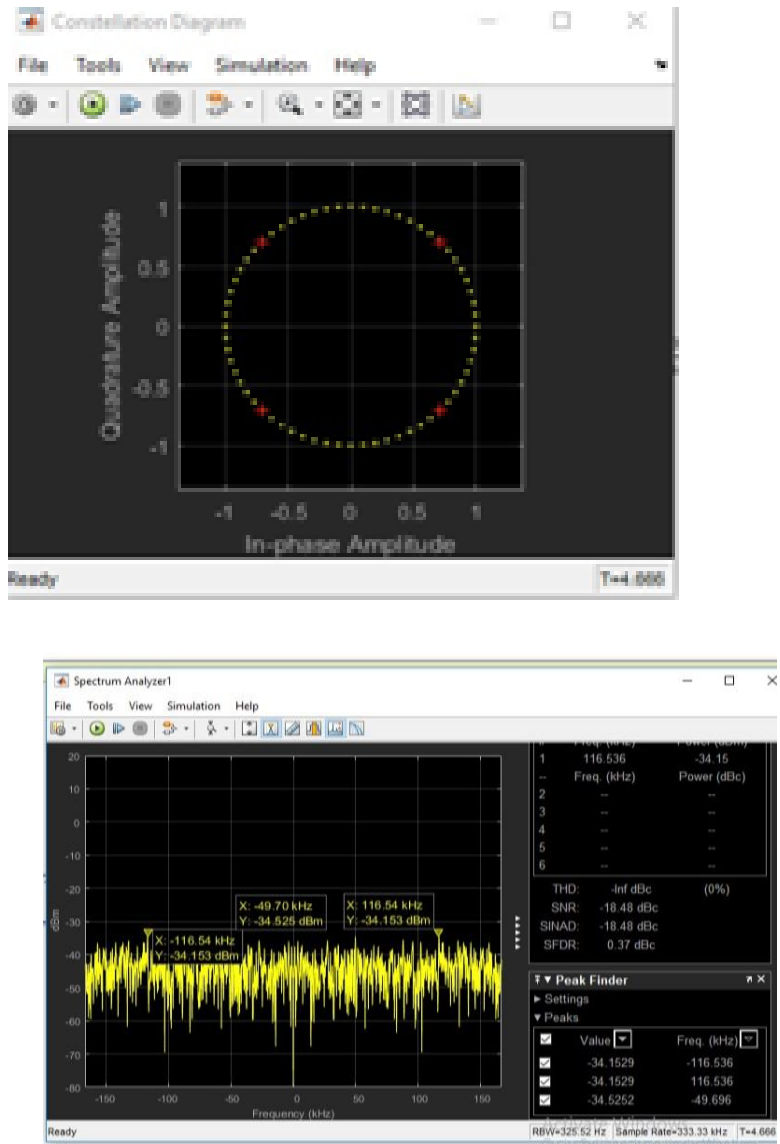

\section{C. bPSK Simulation}
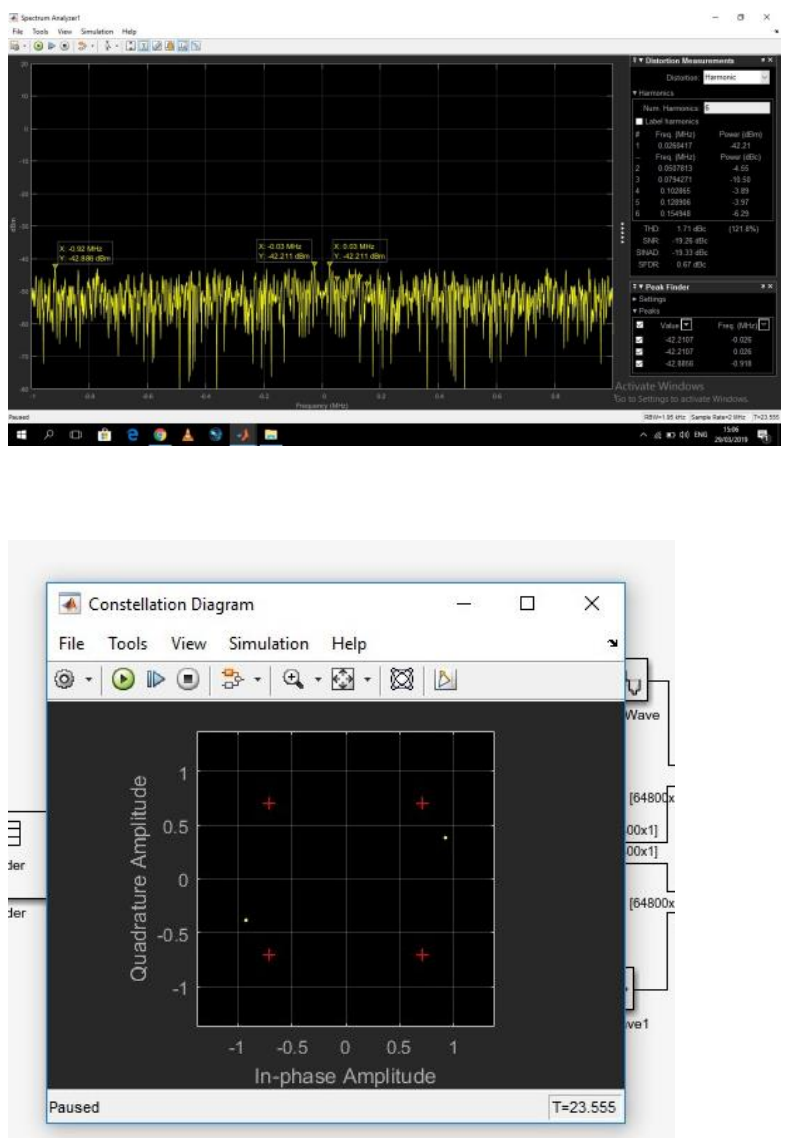

\section{PAPR Block Diagram}

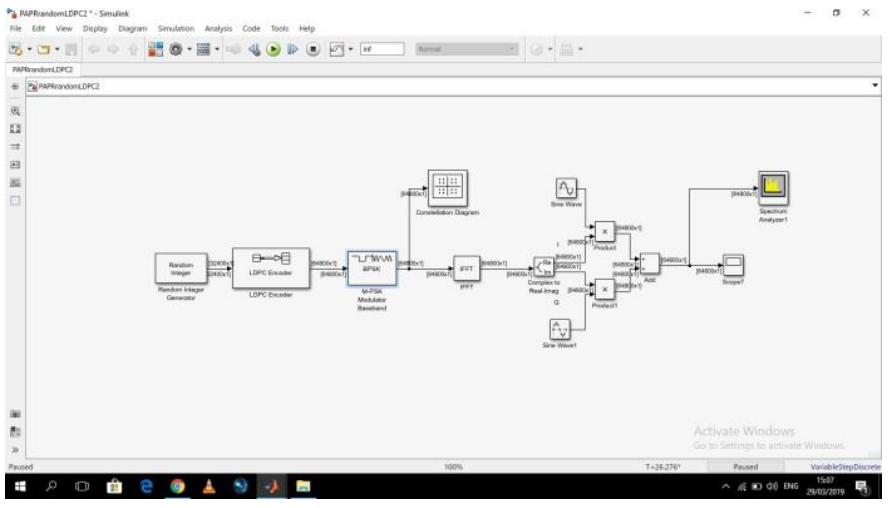

VII. CONCLUSION

In this report the formal assembling of OFDM block with input random bit stream that is coded into LDPC is given to observe the flow of the data through the AWGN channel and received data is checked for good amount of quality with less errors. Signal to noise ratio and Bit error rates were observed in this OFDM system where some comparison of 8 QPSK and 16 QPSK and QAM modulated signals were used. Multi carrier MIMO is often used for $5 \mathrm{G}$ technologies to achieve faster data rates upto 1 GBPS. This work will enhance the knowledge of the $5 \mathrm{G}$ enthusiasts to explore more possibilities of techniques to be incorporated in the project..

\section{FUTURE SCOPE}

It is undoubtedly known that the data speeds are directly impacting the growth of any economy in $21^{\text {st }}$ century

On this note, $5 \mathrm{G}$ wireless communications would bring the life more easy and fast in processing speeds of computations through rapid pace in integrated electronics and millimeter wave technologies together. It is indeed accepted by many research communities that the data transmission technologies will surface the lifestyles very soon with growing interest of sharing the ideas in no time irrespective of time, place \& power. Industry 4.0, Internet of Things (IoT), Internet of Everything (IoE), Terra hertz technologies, Industrial Automation through Artificial Intelligence, Data Science, Data Analytics, Data Mining, Cyber security, Space Colonization, Rocket Science, Satellite Technology, Remote Sensing, Nano-Robotics, Remote Medicine, Intelligent Genetics etc, are some of the most promising technologies for the future century which is only possible with the ease in data transmission that was emphasized in this report.

\section{REFERENCES}

[1] Comprehensive Analysis of UFMC with OFDM and FBMC K. Krishna Kishore1*, P. Rajesh Umar2 and V. Jagan Naveen.

[2] 5G Waveform Candidates by Rohde \& Schwarz., 
[3] Peak to Average Power Ratio (PAPR) reduction in OFDM for a WLAN network using SLM technique, ISBN: 978-1-4244-8679-3, IEEE.

Sathish Kumar Yegireddi M.Tech (Embedded System): Department of ECE,IIIT Srikakulam, RAJIV GANDHI UNIVERSITY OF KNOWLEDGE TECHNOLOGIES(RGUKT),Andhra Pradesh, India

Vemakoti Vivekanand M.Tech, MBA, is a curious researcher in quantum electrodynamics and high speed signal transmissions though had a deep insights on photovoltaic cells' performance calibrations in his previous research papers. He has 6 years of experience in teaching at various engineering and academic institutes, presently working as IIT - Physics lecturer in a national level schooling institute. He is ready to collaborate any level of research stimulus in the aforesaid domains or any promising interdisciplinary prospects of science and technology. 